\title{
Observational constraints on the likelihood of ${ }^{26} \mathrm{Al}$ in planet-forming environments
}

\begin{abstract}
Megan Reiter
UK Astronomy Technology Centre, Blackford Hill, Edinburgh EH9 3HJ, UK

e-mail: megan.reiter@stfc.ac.uk

Received 1 September 2020 / Accepted 17 November 2020

ABSTRACT

Recent work suggests that ${ }^{26} \mathrm{Al}$ may determine the water budget in terrestrial exoplanets as its radioactive decay dehydrates planetesimals leading to rockier compositions. Here I consider the observed distribution of ${ }^{26} \mathrm{Al}$ in the Galaxy and typical star-forming environments to estimate the likelihood of ${ }^{26} \mathrm{Al}$ enrichment during planet formation. I do not assume Solar-System-specific constraints as I am interested in enrichment for exoplanets generally. Observations indicate that high-mass stars dominate the production of ${ }^{26} \mathrm{Al}$ with nearly equal contributions from their winds and supernovae. Observed ${ }^{26} \mathrm{Al}$ abundances are comparable to those in the early Solar System in the high-mass star-forming regions where most stars (and thereby most planets) form. These high abundances appear to be maintained for a few million years, which is much longer than the $0.7 \mathrm{Myr}$ half-life. Observed bulk ${ }^{26} \mathrm{Al}$ velocities are an order of magnitude slower than expected from winds and supernovae. These observations are at odds with typical model assumptions that ${ }^{26} \mathrm{Al}$ is provided instantaneously by high velocity mass loss from supernovae and winds. The regular replenishment of ${ }^{26} \mathrm{Al}$, especially when coupled with the small age differences that are common in high-mass star-forming complexes, may significantly increase the number of star- and planet-forming systems exposed to ${ }^{26} \mathrm{Al}$. Exposure does not imply enrichment, but the order of magnitude slower velocity of ${ }^{26} \mathrm{Al}$ may alter the fraction that is incorporated into planet-forming material. Together, this suggests that the conditions for rocky planet formation are not rare, nor are they ubiquitous, as small regions such as Taurus, that lack high-mass stars to produce ${ }^{26} \mathrm{Al}$ may be less likely to form rocky planets. I conclude with suggested directions for future studies.
\end{abstract}

Key words. planets and satellites: composition - planets and satellites: formation - planets and satellites: terrestrial planets stars: massive - stars: mass-loss - stars: formation

\section{Introduction}

Recent work from Lichtenberg et al. (2019) suggests that the bulk water fraction and radius of terrestrial exoplanets are anticorrelated with the abundance of ${ }^{26} \mathrm{Al}$. Heat released by the radioactive decay of ${ }^{26} \mathrm{Al}$ (half-life $\approx 0.72 \mathrm{Myr}$ ) provides an additional heat source that aids planetesimal differentiation (e.g., Hevey \& Sanders 2006) and dehydrates planetesimals (e.g. Grimm \& McSween 1993), leading to a rockier composition of the final planet.

Whether rocky planets are more conducive to life and detectable bio-signatures is an active area of research (e.g., Kaltenegger 2017). There is particular interest in whether water worlds - those with a significantly higher bulk water fraction than Earth - can support life (e.g., Noack et al. 2016; Kite \& Ford 2018; Olson et al. 2020). Until observations clarify terrestrial exoplanet compositions (e.g., Teske et al. 2018; Rice et al. 2019), models provide the best constraints on the most likely outcomes of planet formation.

The short half-life of ${ }^{26} \mathrm{Al}$ requires production near planet formation in both time and space. Meteoritic evidence indicates that ${ }^{26} \mathrm{Al}$ was abundant in the early Solar System and most enrichment models aim to reproduce this specific case. Early models considered direct supernova $(\mathrm{SN})$ injection into the starforming cloud, perhaps stimulating its collapse (e.g., Cameron $\&$ Truran 1977) and direct enrichment of planet-forming disks (e.g., Chevalier 2000). Recently, attention has turned to preSN mass loss, particularly from Wolf-Rayet (W-R) stars, as a pathway to earlier enrichment (e.g., Gounelle \& Meibom 2008; Gaidos et al. 2009; Dwarkadas et al. 2017). Scenarios involving either $\mathrm{SNe}$ or $\mathrm{W}-\mathrm{R}$ winds require finely-tuned conditions to place young planet-forming systems in close proximity, suggesting that few systems will be formed under their influence.

Extrapolating from the Solar System has led some authors to suggest that the Earth represents a minority $(\sim 1 \%)$ of the outcomes of planet formation (see e.g., Adams 2010; Gounelle 2015; Portegies Zwart 2019). However, the specific conditions inferred for the Solar System may not apply to exoplanets in general. Abundant ${ }^{26} \mathrm{Al}$ in many star- and planet-forming systems would challenge suggestions that water-rich planets may be the dominant family of terrestrial planet analogs (e.g., Alibert \& Benz 2017; Miguel et al. 2020). The importance of ${ }^{26} \mathrm{Al}$ in planet composition clearly warrants a broader assessment of the likelihood of ${ }^{26} \mathrm{Al}$-enrichment.

In this Letter, I compare observations of ${ }^{26} \mathrm{Al}$ in the Galaxy with typical star- and planet-forming conditions to estimate the likelihood of ${ }^{26} \mathrm{Al}$ enrichment. I focus on ${ }^{26} \mathrm{Al}$ because of its critical role in determining the water budget of terrestrial exoplanets. My goal is not to explain the formation of the Earth, so I do not consider other elemental abundances (i.e., ${ }^{60} \mathrm{Fe}$ ) specific to the Solar System. I conclude with a discussion of how models of ${ }^{26} \mathrm{Al}$-enrichment can be improved in light of recent observations. 


\section{Observational constraints}

\subsection{Observations of ${ }^{26} \mathrm{Al}$ and its production by high-mass stars}

Most of our knowledge of the production and distribution of ${ }^{26} \mathrm{Al}$ in the Galaxy comes from observations of the $1.809 \mathrm{MeV}$ $\gamma$-ray photons produced (along with positrons) when it undergoes radioactive decay (Endt 1990). Emission is bright along the Galactic plane corresponding to an average abundance $\sim 3-25$ lower than the early Solar System (Lugaro et al. 2018). However, the emission is predominately associated with young high-mass star-forming regions (e.g., Knödlseder et al. 1999; Diehl et al. 2006). An observed close correlation between $1.809 \mathrm{MeV}$ emission and ionized gas in the interstellar medium (ISM) can only be explained if high-mass stars $(>10)$ dominate ${ }^{26} \mathrm{Al}$ production (Knödlseder et al. 1999).

Classical W-R stars are the late evolutionary phase of highmass stars $\left(M_{\text {initial }} \gtrsim 25 M_{\odot}\right.$ in the Galaxy), and multiple studies argue that their winds contribute $\gtrsim 40 \%$ of the observed ${ }^{26} \mathrm{Al}$ (e.g., Knödlseder et al. 1999). This enhances the ISM abundance of ${ }^{26} \mathrm{Al}$ prior to the onset of supernovae (SNe). Diehl et al. (2010) propose a time-evolution of the ${ }^{26} \mathrm{Al}$ abundance for a given star-forming event as follows: ${ }^{26} \mathrm{Al}$ is initially enhanced in the ISM after $\sim 3$ Myr by the onset of mass loss from W-R winds. Further enhancement comes over the next 1-2 Myr from corecollapse $\mathrm{SNe}$, with the peak value at $\sim 5 \mathrm{Myr}$. The abundance then decreases as lower-mass stars do not reach a W-R phase, making their most significant contribution later from their corecollapse SNe explosions. Beyond 7-8 Myr, the ${ }^{26} \mathrm{Al}$ abundance continues to decrease, with a tail extending to $\sim 20 \mathrm{Myr}$.

Population-synthesis models also point to significant early, pre-supernova enrichment (e.g., Voss et al. 2009). Voss et al. (2010, 2012) modeled the combined high-mass star content of the Orion and Carina star-forming complexes and argue that the current high observed abundance of ${ }^{26} \mathrm{Al}$ has persisted for ¿5 Myr and will be maintained for another $\sim 5 \mathrm{Myr}$ with an additional enhancement from $\mathrm{SNe}$.

Doppler shifts of the $1.809 \mathrm{MeV}$ line indicate that ${ }^{26} \mathrm{Al}-$ producing sources corotate with the Galaxy (Diehl et al. 2006). However, the bulk velocity of ${ }^{26} \mathrm{Al}$-enriched material is higher than that of the molecular gas in the Galaxy, with a systematic offset of $\sim 200 \mathrm{~km} \mathrm{~s}^{-1}$ in the direction of Galactic rotation (Kretschmer et al. 2013). In the specific case of Sco OB2, ${ }^{26} \mathrm{Al}$ was detected with slightly blueshifted energies, suggesting a bulk velocity of $137 \pm 75 \mathrm{~km} \mathrm{~s}^{-1}$ (Diehl et al. 2010). These velocities are roughly an order of magnitude slower than the expected ejection velocities $\left(>1000 \mathrm{~km} \mathrm{~s}^{-1}\right)$ for winds and $\mathrm{SNe}$.

\subsection{Typical star-forming conditions}

Observations in the Milky Way and in other galaxies suggest a cluster mass function of the form $\mathrm{d} N / \mathrm{d} M \sim M^{-2}$ (e.g., Mok et al. 2020). I refer to clustering in the statistical sense that stars form near other stars (see discussion in Lee \& Hopkins 2020), noting that many extra-galactic studies do not apply additional criteria to determine whether stellar over-densities are gravitationally bound. As Dukes \& Krumholz (2012) point out, this cluster mass function implies that $\gtrsim 50 \%$ of stars are born in high-mass starforming regions with as many or more high-mass stars than the Orion Nebula Cluster (ONC).

The Orion star-forming complex is nevertheless observed to have ${ }^{26} \mathrm{Al}$ (Diehl et al. 2003) at a level similar to the early Solar System (Jura et al. 2013). The same is true of Carina (Reiter \& Parker 2019). Jura et al. (2013) also make a more general argument by comparing the Galactic ${ }^{26} \mathrm{Al}$ abundance with molecular (star-forming) gas to show that star- and planetformation regions have abundances on the order of the early Solar System, unlike the average ISM.

\section{Discussion}

The majority of stars ( $\gtrsim 50 \%$ ) form in high-mass regions where ${ }^{26} \mathrm{Al}$ is observed to be abundant. While this suggests that many systems are exposed to ${ }^{26} \mathrm{Al}$, two key uncertainties affect the fraction of systems that are ultimately enriched: (1) when and how long ${ }^{26} \mathrm{Al}$ is highly abundant and (2) how and whether the ${ }^{26} \mathrm{Al}$ mixes with the star- and planet-forming material. I discuss these in turn below.

\subsection{The timescale of ${ }^{26} \mathrm{Al}$ availability}

Observations of ${ }^{26} \mathrm{Al}$ indicate a fundamental discrepancy with typical model assumptions: high ${ }^{26} \mathrm{Al}$ abundances appear to persist for a few million years, which is much longer than the 0.7 Myr half-life assumed for an instantaneous production event. The longer timescale reflects production by a stellar population rather than a single high-mass source.

For models that assume a single-age stellar population, disks must survive long enough for direct pollution from a nearby SN or W-R star. Prior to these late evolutionary stages, feedback from those same high-mass stars may accelerate disk destruction, limiting the number of remaining systems to enrich (e.g., Winter et al. 2018; Concha-Ramírez et al. 2019).

Other models start with a SN or W-R winds polluting nearby cold gas that will subsequently form a second (or third) generation of stars (e.g., Gaidos et al. 2009; Gounelle \& Meynet 2012; Kuffmeier et al. 2016; Dwarkadas et al. 2017). However, several studies show that most of the gas is cleared from starforming regions by pre-SN feedback (e.g., Hollyhead et al. 2015; Chevance et al. 2019).

Reality likely lies between these scenarios. Observations consistently suggest small age spreads in star-forming regions (e.g., Getman et al. 2014; Beccari et al. 2017; Venuti et al. 2018; Jerabkova et al. 2019; Prisinzano et al. 2019). Age differences where younger and older populations are not necessarily spatially coincident - are also common within OB associations (e.g., Wright et al. 2010; Pecaut \& Mamajek 2016; Getman et al. 2018). In both cases, age differences are a few million years, which is much smaller than would be produced by triggered sequential star formation (Parker \& Dale 2016).

Age differences affect ${ }^{26} \mathrm{Al}$-enrichment in two ways: (1) they extend the time that disks are in the region (as in the proplyd lifetime solution proposed by Winter et al. 2019); and (2) they provide regular replenishment to maintain the high abundance of ${ }^{26} \mathrm{Al}$ for several million years. This pragmatic approach is more akin to the hierarchical star formation models from Fujimoto et al. (2018), which show that galactic-scale correlations in star formation strongly affect enrichment.

The Carina star-forming complex offers an illustrative example. Several studies suggest age differences between the primary clusters $\operatorname{Tr} 14, \operatorname{Tr} 15$, and $\operatorname{Tr} 16$ (see, e.g., the discussion in Townsley et al. 2011 for a review). Recent work from Povich et al. (2019) quantifies variations in the duration of star formation in Carina. They conclude that star formation has been ongoing for $\sim 10 \mathrm{Myr}$, with a peak in the star-formation rate $\sim 3 \mathrm{Myr}$ ago with the birth of the famous central clusters, $\operatorname{Tr} 14$ and $\operatorname{Tr} 16$.

These multiple episodes of star formation are all contained within the $\gtrsim 1^{\circ}$ beamsize of the $\gamma$-ray observations. Voss et al. (2012) performed population synthesis modeling of 
the combined population of all clusters to estimate that the current high abundance has been maintained for $\sim 5 \mathrm{Myr}$ and will persist for another $\sim 5 \mathrm{Myr}$.

If ${ }^{26} \mathrm{Al}$ abundances have been maintained at the current high levels for $\sim 5 \mathrm{Myr}$ while star formation peaked within the last $\sim 3 \mathrm{Myr}$, then the majority of systems were likely exposed to ${ }^{26} \mathrm{Al}$. Similar arguments can be made for the Orion star-forming complex. If $\gtrsim 50 \%$ of all stars are born in high-mass regions with (conservatively) half of those exposed to significant ${ }^{26} \mathrm{Al}$, then we may crudely estimate that as many as $\sim 25 \%$ of systems may be enriched with ${ }^{26} \mathrm{Al}$, possibly at a level adequate to make rocky planets. This is an order of magnitude larger than the few percent estimated for Solar-System-specific conditions, but is consistent with model results from Fujimoto et al. (2018) that short-lived radioactive isotopes, including ${ }^{26} \mathrm{Al}$, are abundant in newly formed stars.

This order-of-magnitude estimate does not account for uncertainties in the cluster mass function and ignores possible variations with location in a galaxy. No provision is made for differences in ${ }^{26} \mathrm{Al}$ production with stellar mass, although not all high-mass stars undergo a W-R phase and SN yields depend on the mass of the progenitor. More quantitative analysis is clearly needed. I outline some suggested directions in Sect. 3.4.

\subsection{The incorporation of ${ }^{26} \mathrm{Al}$ into planet-forming material}

Exposure to ${ }^{26} \mathrm{Al}$ does not imply that it mixes with planetforming material. Here too, observations invite the reconsideration of typical model assumptions. The observed bulk velocity of the ${ }^{26} \mathrm{Al}$ is $\sim 200 \mathrm{~km} \mathrm{~s}^{-1}$, which is an order of magnitude slower than the $\sim 1000 \mathrm{~km} \mathrm{~s}^{-1}$ expected from W-R winds or SNe (see Sect. 2.1). Slower ${ }^{26} \mathrm{Al}$ may mix more readily with planetforming material. Existing parameter studies of disk enrichment investigate only a narrow range of velocities that are much faster than the observed bulk ${ }^{26} \mathrm{Al}$ velocity (e.g., Ouellette et al. 2007).

Other models for the enrichment of star- and planet-forming gas enforce mixing by confining ${ }^{26} \mathrm{Al}$-enriched ejecta (e.g., Vasileiadis et al. 2013; Gounelle 2015; Kuffmeier et al. 2016). This would preclude ${ }^{26} \mathrm{Al}$-enrichment in regions such as Carina that have developed into superbubbles that are unable to contain ${ }^{26} \mathrm{Al}$-enriched material. As pointed out by Fujimoto et al. (2018), these scenarios are at odds with both the short observed time for young high-mass star-forming regions to clear their gas and the large observed scale height of ${ }^{26} \mathrm{Al}$ in the Galaxy (Wang et al. 2009).

While the physical pathway by which ${ }^{26} \mathrm{Al}$ is incorporated into planet-forming material remains unclear, composition studies of extrasolar asteroids suggest that mixing, nevertheless, does occur. Jura et al. (2013) estimated the ${ }^{26} \mathrm{Al}$ required to enable the observed differentiation and find that it is comparable to the ${ }^{26} \mathrm{Al}$ abundance in Orion, implying that Solar System levels of enrichment are common.

Additional evidence may come from the observed size distribution of exoplanets. The models of Lichtenberg et al. (2019) predict that the planet radius is anticorrelated with the bulk water fraction. Observations of small, close-in exoplanets represent a bimodal radius distribution with current data suggesting the same intrinsic frequency for the two regimes (Fulton et al. 2017).

\subsection{Some environments are more likely to form rocky planets}

The points in the previous sections suggest that some starforming environments will be more conducive than others to
${ }^{26} \mathrm{Al}$-enrichment, and thus rocky planet formation. The most massive member of Taurus, the local template of a low-mass star-forming region, is $\sim 3.5 M_{\odot}$ (Table 1, De Marchi et al. 2010). Such a star will end its life as an AGB star that will not sustain temperatures sufficient to synthesize ${ }^{26} \mathrm{Al}$ (e.g., Abia et al. 2017). If ${ }^{26} \mathrm{Al}$ regulates the water budget, as in the models of Lichtenberg et al. (2019), this suggests that terrestrial planets that form in low-mass regions such as Taurus are more likely to be water worlds.

\subsection{Future directions}

In the following sections, I highlight key areas for future work to quantify the fraction of systems that are enriched with ${ }^{26} \mathrm{Al}$ and, therefore, able to form rocky planets.

\subsubsection{Production of ${ }^{26} \mathrm{Al}$ by high-mass stars}

Stellar mass is only one of the relevant variables affecting the timescale and abundance of ${ }^{26} \mathrm{Al}$ production. Rotation is a key parameter that influences the amount of ${ }^{26} \mathrm{Al}$ introduced into the ISM by high-mass stars, primarily via rotational-mixing of material from deeper in the star to the surface (e.g., Voss et al. 2009). By bringing ${ }^{26} \mathrm{Al}$ to the surface earlier than in models without rotation, stars can potentially return ${ }^{26} \mathrm{Al}$ to the ISM via their stellar winds both earlier and for lower initial masses than in nonrotating models. For ${ }^{26} \mathrm{Al}$-enrichment, the effects of rotation have been explored for initial rotational velocities of $300 \mathrm{~km} \mathrm{~s}^{-1}$ (e.g., Palacios et al. 2005; Voss et al. 2009).

Surveys such as the VLT-FLAMES Tarantula Survey (Evans et al. 2011) and the IACOB project (Simón-Díaz et al. 2011a,b, 2015) have provided large spectroscopic samples to investigate the rotation rates of high-mass stars (e.g., Ramírez-Agudelo et al. 2013; Holgado et al. 2018; Holgado 2019). Typical rotation rates are less than half of those assumed in population synthesis studies (e.g., Voss et al. 2009). At these velocities, stellar evolution more closely resembles nonrotating models (Evans et al. 2020). Existing population synthesis models matched the observed ${ }^{26} \mathrm{Al}$, so it is unclear if changes to ${ }^{26} \mathrm{Al}$ production also affect the time-evolution of the abundance.

Another critical development in the last 10 years is evidence that $>70 \%$ of high-mass stars are in close-separation binaries that will eventually interact (Sana et al. 2012). Large grids of binary models are only now being computed. Recent work from Brinkman et al. (2019) presents non-rotating models that suggest that binary interactions affect the total ${ }^{26} \mathrm{Al}$ mass ejected by only $\sim 5-10 \%$. There is a substantial enhancement of ${ }^{26} \mathrm{Al}$ from $10-15 M_{\odot}$ primaries in binary systems, but the total amount from a given population is dominated by higher mass stars (>30 $M_{\odot}$ ), where binary effects appear to be less important. Further work is required to investigate the impact of stellar rotation on such models.

\subsubsection{Pre-supernova enrichment models}

Studies of the $\gamma$-ray emission from the radioactive decay of ${ }^{26} \mathrm{Al}$ point to the important role of pre-SN mass loss. Including the pre-SN enrichment in cluster-scale simulations (e.g., Lichtenberg et al. 2016; Nicholson et al. 2019) may significantly change the fraction of disks enriched with ${ }^{26} \mathrm{Al}$. Models should also consider on-going ${ }^{26} \mathrm{Al}$ production, and perhaps steady accretion instead of a single injection event. Meteoritic evidence suggests this may have happened in the Solar System with ${ }^{26} \mathrm{Al}$ accrued quickly, but not instantaneously (Liu et al. 2012). 


\section{Summary}

Recent models suggest that the ${ }^{26} \mathrm{Al}$ abundance plays a central role in determining the water budget of terrestrial planets. Galactic observations of the short-lived radioactive isotope ${ }^{26} \mathrm{Al}$ provide a constraint on the fraction of star- and planetforming systems that may be enriched. Observations indicate that ${ }^{26} \mathrm{Al}$ is predominantly produced by high-mass stars. Pre-SN mass loss, especially from W-R stars, is thought to contribute nearly half of the Galactic ${ }^{26} \mathrm{Al}$ budget. Furthermore, ${ }^{26} \mathrm{Al}$ is particularly abundant in high-mass star-forming regions where the majority of stars, and thus planets, form. Mass loss by stellar winds can enrich the ISM in such regions in a few million years. Critically for planet formation, the regular replenishment may sustain high levels of ${ }^{26} \mathrm{Al}$ for millions of years (much longer than its $0.7 \mathrm{Myr}$ half-life). I argue that the majority of star and planet systems in high-mass star-forming regions may be exposed to abundant ${ }^{26} \mathrm{Al}$. Exposure does not imply enrichment, but observed bulk ${ }^{26} \mathrm{Al}$ velocities are an order of magnitude slower than expected from winds and $\mathrm{SNe}$, which may increase the likelihood of enrichment. If half of the exposed systems are enriched with ${ }^{26} \mathrm{Al}$, this (rough) estimate suggests that the fraction of such systems is at least an order of magnitude higher than the few percent found by studies that extrapolate from Solar-System-specific conditions. More quantitative estimates that do not assume Solar-System-specific conditions a priori are clearly needed to clarify this fraction.

These conditions describe the environment in which most stars and planets form, but they do not reflect those sampled in low-mass star-forming clouds. This suggests that rocky planets are more likely to form around stars born in high-mass star-forming regions. Low-mass regions such as Taurus do not contain the high-mass stars that dominate the production of ${ }^{26} \mathrm{Al}$ and thus are unlikely to form rocky planets.

Acknowledgements. I would like to thank the referee for a timely and thoughtful report that improved the manuscript. I am deeply grateful to Chris Evans, Richard Parker, Ken Rice, and Tim Lichtenberg for reading the manuscript and providing thoughtful comments. Thanks also to Brandt Gaches and Karen Moran. This project received funding from the European Union's Horizon 2020 research and innovation programme under the Marie Skĺodowska-Curie grant agreement No. 665593 awarded to the Science and Technology Facilities Council.

\section{References}

Abia, C., Hedrosa, R. P., Domínguez, I., \& Straniero, O. 2017, A\&A, 599, A39

Adams, F. C. 2010, ARA\&A, 48, 47

Alibert, Y., \& Benz, W. 2017, A\&A, 598, L5

Beccari, G., Petr-Gotzens, M. G., Boffin, H. M. J., et al. 2017, A\&A, 604, A22

Brinkman, H. E., Doherty, C. L., Pols, O. R., et al. 2019, ApJ, 884, 38

Cameron, A. G. W., \& Truran, J. W. 1977, Icarus, 30, 447

Chevalier, R. A. 2000, ApJ, 538, L151

Chevance, M., Kruijssen, J. M. D., Hygate, A. P. S., et al. 2019, MNRAS, 493 , 2872

Concha-Ramírez, F., Wilhelm, M. J. C., Portegies Zwart, S., \& Haworth, T. J. 2019, MNRAS, 490, 5678

De Marchi, G., Paresce, F., \& Portegies Zwart, S. 2010, ApJ, 718, 105

Diehl, R., Kretschmer, K., Plüschke, S., et al. 2003, Astron. Nachr., 324, 18

Diehl, R., Halloin, H., Kretschmer, K., et al. 2006, Nature, 439, 45
Diehl, R., Lang, M. G., Martin, P., et al. 2010, A\&A, 522, A51

Dukes, D., \& Krumholz, M. R. 2012, ApJ, 754, 56

Dwarkadas, V. V., Dauphas, N., Meyer, B., Boyajian, P., \& Bojazi, M. 2017, ApJ, 851,147

Endt, P. M. 1990, Nucl. Phys. A, 521, 1

Evans, C. J., Taylor, W. D., Hénault-Brunet, V., et al. 2011, A\&A, 530, A108

Evans, C., Taylor, W., Sana, H., et al. 2020, The Messenger, 181, 22

Fujimoto, Y., Krumholz, M. R., \& Tachibana, S. 2018, MNRAS, 480, 4025

Fulton, B. J., Petigura, E. A., Howard, A. W., et al. 2017, AJ, 154, 109

Gaidos, E., Krot, A. N., Williams, J. P., \& Raymond, S. N. 2009, ApJ, 696, 1854

Getman, K. V., Feigelson, E. D., Kuhn, M. A., et al. 2014, ApJ, 787, 108

Getman, K. V., Feigelson, E. D., Kuhn, M. A., et al. 2018, MNRAS, 476, 1213

Gounelle, M. 2015, A\&A, 582, A26

Gounelle, M., \& Meibom, A. 2008, ApJ, 680, 781

Gounelle, M., \& Meynet, G. 2012, A\&A, 545, A4

Grimm, R. E., \& McSween, H. Y. 1993, Science, 259, 653

Hevey, P. J., \& Sanders, I. S. 2006, Meteor. Planet. Sci., 41, 95

Holgado, G. 2019, PhD Thesis, University of La Laguna, Spain

Holgado, G., Simón-Díaz, S., Barbá, R. H., et al. 2018, A\&A, 613, A65

Hollyhead, K., Bastian, N., Adamo, A., et al. 2015, MNRAS, 449, 1106

Jerabkova, T., Beccari, G., Boffin, H. M. J., et al. 2019, A\&A, 627, A57

Jura, M., Xu, S., \& Young, E. D. 2013, ApJ, 775, L41

Kaltenegger, L. 2017, ARA\&A, 55, 433

Kite, E. S., \& Ford, E. B. 2018, ApJ, 864, 75

Knödlseder, J., Bennett, K., Bloemen, H., et al. 1999, A\&A, 344, 68

Kretschmer, K., Diehl, R., Krause, M., et al. 2013, A\&A, 559, A99

Kuffmeier, M., Frostholm Mogensen, T., Haugbølle, T., Bizzarro, M., \& Nordlund, A. 2016, ApJ, 826, 22

Lee, E. J., \& Hopkins, P. F. 2020, MNRAS, 495, L86

Lichtenberg, T., Parker, R. J., \& Meyer, M. R. 2016, MNRAS, 462, 3979

Lichtenberg, T., Golabek, G. J., Burn, R., et al. 2019, Nat. Astron., 3, 307

Liu, M.-C., Chaussidon, M., Göpel, C., \& Lee, T. 2012, Earth Planet. Sci. Lett., 327,75

Lugaro, M., Ott, U., \& Kereszturi, Á. 2018, Prog. Part. Nucl. Phys., 102, 1

Miguel, Y., Cridland, A., Ormel, C. W., Fortney, J. J., \& Ida, S. 2020, MNRAS, 491, 1998

Mok, A., Chandar, R., \& Fall, S. M. 2020, ApJ, 893, 135

Nicholson, R. B., Parker, R. J., Church, R. P., et al. 2019, MNRAS, 485, 4893

Noack, L., Höning, D., Rivoldini, A., et al. 2016, Icarus, 277, 215

Olson, S. L., Jansen, M., \& Abbot, D. S. 2020, ApJ, 895, 19

Ouellette, N., Desch, S. J., \& Hester, J. J. 2007, ApJ, 662, 1268

Palacios, A., Meynet, G., Vuissoz, C., et al. 2005, A\&A, 429, 613

Parker, R. J., \& Dale, J. E. 2016, MNRAS, 456, 1066

Pecaut, M. J., \& Mamajek, E. E. 2016, MNRAS, 461, 794

Portegies Zwart, S. 2019, A\&A, 622, A69

Povich, M. S., Maldonado, J. T., Haze Nuñez, E., \& Robitaille, T. P. 2019, ApJ, 881,37

Prisinzano, L., Damiani, F., Kalari, V., et al. 2019, A\&A, 623, A159

Ramírez-Agudelo, O. H., Simón-Díaz, S., Sana, H., et al. 2013, A\&A, 560, A29

Reiter, M., \& Parker, R. J. 2019, MNRAS, 486, 4354

Rice, K., Malavolta, L., Mayo, A., et al. 2019, MNRAS, 484, 3731

Sana, H., de Mink, S. E., de Koter, A., et al. 2012, Science, 337, 444

Simón-Díaz, S., Garcia, M., Herrero, A., Maíz Apellániz, J., \& Negueruela, I. 2011a, Stellar Clusters \& Associations: A RIA Workshop on Gaia, 255

Simón-Díaz, S., Castro, N., Garcia, M., Herrero, A., \& Markova, N. 2011 b, Bull. Soc. R. Sci. Liege, 80, 514

Simón-Díaz, S., Negueruela, I., Maíz Apellániz, J., et al. 2015, Highlights of Spanish Astrophysics VIII, 576

Teske, J. K., Wang, S., Wolfgang, A., et al. 2018, AJ, 155, 148

Townsley, L. K., Broos, P. S., Corcoran, M. F., et al. 2011, ApJS, 194, 1

Vasileiadis, A., Nordlund, A., \& Bizzarro, M. 2013, ApJ, 769, L8

Venuti, L., Prisinzano, L., Sacco, G. G., et al. 2018, A\&A, 609, A10

Voss, R., Diehl, R., Hartmann, D. H., et al. 2009, A\&A, 504, 531

Voss, R., Diehl, R., Vink, J. S., \& Hartmann, D. H. 2010, A\&A, 520, A51

Voss, R., Martin, P., Diehl, R., et al. 2012, A\&A, 539, A66

Wang, W., Lang, M. G., Diehl, R., et al. 2009, A\&A, 496, 713

Winter, A. J., Clarke, C. J., Rosotti, G., et al. 2018, MNRAS, 478, 2700

Winter, A. J., Clarke, C. J., Rosotti, G. P., Hacar, A., \& Alexander, R. 2019,

MNRAS, 490, 5478

Wright, N. J., Drake, J. J., Drew, J. E., \& Vink, J. S. 2010, ApJ, 713, 871 